\title{
COMPARISON OF NEONATAL GASTROINTESTINAL PERFORATION DUE TO NECROTIZING ENTEROCOLITIS AND OTHER CAUSES
}

\author{
S.-L. Lee ${ }^{1}$, K.-J. Kang ${ }^{1}$, C.-S. Kim ${ }^{1}$, S.-O. Choi ${ }^{2}$, W.-H. Park ${ }^{2}$ \\ ${ }^{1}$ Pediatrics, ${ }^{2}$ Pediatric Surgery, Dongsan Medical Center, Keimyung University, Daegu, Republic of Korea
}

Purpose: Gastrointestinal perforation is one of the serious diseases in neonatal period. We compared the clinical features and outcome for neonatal gastrointestinal perforation due to necrotizing enterocolitis (NEC) and other etiologic diseases (non-NEC).

Methods: A retrospective review of medical records of neonates with gastrointestinal perforation admitted to the neonatal intensive care unit of Dongsan Medical Center between January 1999 and December 2009 was enrolled. Admission records for gestational age, day of perforation, type of delivery and mortality rate were reviewed and statistically analyzed for both groups.

Results: Among 28 neonates, NEC group was 10 cases (35.7\%), other diseases were intestinal atresia 25\%, malrotation $10.7 \%$, meconium peritonitis $10.7 \%$, spontaneous perforation $7.1 \%$. The mean gestational age was significantly shorter in the NEC group (32.8 weeks) than in the non-NEC group (36.8 weeks) $(P=0.028)$, and the mean day of perforation was significantly later in the NEC group (16.2 days of life) than in the nonNEC group (2.2 days of life) $(P=0.001)$. The mortality rate was markedly higher in the NEC group (50\%) than in the non-NEC group (5.6\%) $(P=0.013)$. After logistic regression analysis, NEC group itself (OR 7.7, $95 \%$ CI: $0.6-108.1, P=0.130)$ did not increase the mortality rate significantly.

Conculsion: Our study suggests that neonatal gastrointestinal perforation in the NEC group occured later and had a higher mortality rate than the non-NEC group. But, after logistic analysis, NEC itself did not increase the mortality rate significantly. 